\title{
Controlled formation of metastable germanium polymorphs
}

\author{
Bianca Haberl, ${ }^{1, *}$ Malcolm Guthrie, ${ }^{2}$ Brad D. Malone, ${ }^{3}$ Jesse S. Smith, ${ }^{4}$ Stanislav V. Sinogeikin, ${ }^{4}$ Marvin L. Cohen,, 6 \\ James S. Williams, ${ }^{1}$ Guoyin Shen, ${ }^{4}$ and Jodie E. Bradby ${ }^{1}$ \\ ${ }^{1}$ Department of Electronic Materials Engineering, Research School of Physics and Engineering, Australian National University, \\ Canberra ACT 0200, Australia \\ ${ }^{2}$ Geophysical Laboratory, Carnegie Institution of Washington, Washington, DC 20015, USA \\ ${ }^{3}$ School of Engineering and Applied Sciences, Harvard University, Cambridge, Massachusetts 02138, USA \\ ${ }^{4}$ High Pressure Collaborative Access Team, Geophysical Laboratory, Carnegie Institution of Washington, Argonne, Illinois 60439, USA \\ ${ }^{5}$ Department of Physics, University of California, Berkeley, California 94720, USA \\ ${ }^{6}$ Material Sciences Division, Lawrence Berkeley National Laboratory, Berkeley, California 94720, USA
}

(Received 13 February 2014; revised manuscript received 1 April 2014; published 25 April 2014)

\begin{abstract}
The nucleation of metastable germanium polymorphs on decompression is studied using in situ synchrotron $\mathrm{x}$ ray diffraction. We show that the transition pathway is critically dependent on the hydrostaticity. Quasihydrostatic conditions result in the nucleation of the rhombohedral $r 8$ phase, followed by the cubic bc 8 and hexagonal diamond phases. In contrast, the presence of shear yields the tetragonal st12 phase. Thus, targeted nucleation of a metastable polymorph is now possible. This observation has implications for the technological exploitation of $\mathrm{Ge}$, but also for other tetrahedral systems.
\end{abstract}

DOI: 10.1103/PhysRevB.89.144111

PACS number(s): 64.60.My, 61.05.cp, 64.70.kg, 81.30.Dz

\section{INTRODUCTION}

The extensive polymorphism of the group IV results in a variety of metastable polymorphs [1-8] with very different characteristics from their lowest energy structures. For silicon and germanium, these polymorphs exhibit attractive electronic and optical properties [9-14] and may, through doping, allow for superconductivity [11]. Under pressure the diamondcubic (dc) $\mathrm{Si}$ and $\mathrm{Ge}$ (space group $F d \overline{3} m$ ) transform to the metallic white tin structure, $(\beta-\mathrm{Sn}),\left(I 4_{1} /\right.$ amd $)$ at $\sim 10 \mathrm{GPa}$, $[15,16]$. Upon further compression, several reversible metalmetal transitions follow [17]. On decompression, the $(\beta-\mathrm{Sn})$ structure is kinetically unable to transform back to the dc phase. Instead, metastable polymorphs with body-

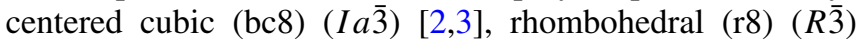
[7], lonsdaleite/hexagonal diamond (hd) $\left(P 6_{3} / m m c\right)$ [2,4], or simple tetragonal (st12) structures $\left(P 4_{3} 2{ }_{1} 2\right)$ [1,18] are formed [19].

This metastable decompression regime exhibits distinct differences between $\mathrm{Si}$ and $\mathrm{Ge}$. Upon decompression from $(\beta-\mathrm{Sn})-\mathrm{Si}, \mathrm{bc} 8-\mathrm{Si}[2,20]$ is formed via r8-Si [7]. bc8-Si then transforms to hd-Si upon heating to $200{ }^{\circ} \mathrm{C}$ [2,21]. Matters are more complex for $\mathrm{Ge}$, where most studies report st12-Ge after unloading $[1,4,6,18,20,22]$. Only upon very fast unloading or unloading at low temperatures has bc8-Ge been reported $[3,6,23,24]$. This latter is unstable at room temperature and anneals fully to hd-Ge within hours $[3,6,21,25]$. In contrast, after pressure-induced metallization of amorphous $\mathrm{Ge}$, the phase upon decompression is more often bc8-Ge [22,25,26] than st12-Ge [27], independent of rate and temperature. Furthermore, the comparison with $\mathrm{Si}$ suggests a pure r8 intermediate should exist between ( $\beta$-Sn)-Ge and bc8-Ge, but this has not been reported to date. Instead, $r 8-G e$ has only been observed after indentation [26], at low temperatures [23], in combination with dc-Ge and st12-Ge [18,28], or

\footnotetext{
*bianca.haberl@gmail.com
}

containing impurities when formed from clathrates [28]. Thus, the transition pathways in Ge are poorly understood, preventing the targeted formation and detailed characterization of specific metastable polymorphs.

In this paper, we show that the Ge transition pathway depends strongly on the hydrostaticity and is independent of decompression rate. We also report the formation of a pure r8-Ge intermediate in a diamond anvil cell (DAC). Therefore, samples of pure crystalline Ge were compressed in a DAC to $(\beta-\mathrm{Sn})-\mathrm{Ge}$. Then pressure was released at different rates. We found that quasihydrostatic pressure always resulted in pure r8-Ge, followed by bc8-Ge. Small amounts of shear, however, were observed to push Ge into the st12 transition pathway without any evidence of the $\mathrm{r} 8 / \mathrm{bc} 8$ phases. Thus, our study now enables controlled formation of specific metastable polymorphs solely by control of shear.

\section{EXPERIMENTAL DETAILS}

Crystalline Ge was loaded into "symmetric"-design DACs equipped with diamond anvils (300 and $400 \mu \mathrm{m}$ culets) and rhenium gaskets (chambers of $\sim 150 \mu \mathrm{m}$ diameter, $\sim 50 \mu \mathrm{m}$ height and $\sim 200 \mu \mathrm{m}$ diameter, $\sim 70 \mu \mathrm{m}$ height). For optimal hydrostatic conditions, a small flake of compressed 100-mesh Ge powder was loaded into the cell, ensuring it did not touch the walls of the chamber nor bridge the diamonds. A ruby ball and gold powder were added as in situ pressure calibrants, also without bridging the anvils. Loading with neon gas as the pressure medium was performed at GSECARS, Advanced Photon Source, Argonne National Laboratory. For high shear stresses another DAC was completely filled with Ge powder without any pressure medium.

In situ powder X-ray diffraction was performed at HPCAT, Advanced Photon Source, Argonne National Laboratory, using $20.000 \mathrm{keV}$ monochromatic $\mathrm{x}$ rays $(\lambda=0.6199 \AA)$ of an insertion-device beamline (16 ID-B). Pressure was applied and released using HPCAT's membrane pressure control system [29]. Two-dimensional (2D) diffraction patterns were collected 
continuously on the Pilatus $1 \mathrm{M}-\mathrm{F}$ detector and reduced to one-dimensional (1D) profiles using FIT2D [30,31].

During decompression, pressure was determined by the unit cell volume of gold and an equation of state [32]. This consistently yielded an apparent negative pressure $(\sim 0.5 \mathrm{GPa})$ after full decompression, suggesting an absolute error of this order. However, the relative error is only limited by the accuracy of the determination of the (111)Au position $( \pm 0.001 \AA)$, thus giving a precision of $\pm 0.05 \mathrm{GPa}$.

Metallic $(\beta-\mathrm{Sn})$-Ge was created by compressing to $\sim 15-$ $18 \mathrm{GPa}$ using a first membrane system, with a second membrane system used to facilitate unloading. In the quasihydrostatic cases, three different sample loadings and cells were used for four different unloading times. For consistency, they are quoted from $10 \mathrm{GPa}$ downward and were $\sim 0.3 \mathrm{~s}$ for decompression to ambient pressure (very fast), $\sim 69 \mathrm{~s}$ to $3 \mathrm{GPa}$ (fast), $\sim 390 \mathrm{~s}$ to ambient pressure (intermediate), and $\sim 3150 \mathrm{~s}$ to ambient pressure (slow). Acquisition times of $0.01,0.1,1$, and $10 \mathrm{~s}$, respectively, were used. In all cases, a readout time of $0.003 \mathrm{~s}$ is included. No full decompression was performed in the fast case, since this sample was remetallized and consequently used for the intermediate decompression. The very fast and slow decompression were performed on two further samples. In the shear case, only fast unloading was employed, from $10 \mathrm{GPa}$ to ambient pressure within $\sim 80 \mathrm{~s}$ with $1 \mathrm{~s}$ acquisition time. Such a full run is shown in Fig. 1(a), where all continuously collected 1D spectra during very fast decompression are displayed. The phase evolution is depicted with time since transitions continue after full decompression and since pressure is nonlinear with respect to time. The latter is presumably due to the large volume differences between different Ge phases.

\section{RESULTS}

Upon unloading in quasihydrostatic conditions, a transition from ( $\beta$-Sn)-Ge to r8-Ge is always observed, fully independent of rate. 1D x-ray diffraction spectra of the $\mathrm{r} 8$ structure at $\sim 4 \mathrm{GPa}$ for all unloading times are shown in Fig. 1(c). r8-Ge was refined with the $R \overline{3}$ space group (structurally equivalent to r8-Si) using EXPGUI [33] and GSAS [34]. In the very fast and fast cases, significant preferred orientation was observed in the 2D patterns and a LeBail approach [35] was used in refinement, while a full Rietveld was performed in the other two cases. In all cases, refinement gave very similar lattice parameters at $\sim 4 \mathrm{GPa}, a=5.9102, \gamma=110.00$ (very fast), $a=5.9069, \quad \gamma=110.05$ (fast), $a=5.9179, \quad \gamma=110.06$ (intermediate), and $a=5.9130, \gamma=110.03$ (slow). These consistent lattice parameters indicate that no significant heat evolved during unloading.

Upon further decompression, r8-Ge transforms to bc8-Ge in the same manner as r8-Si does to bc8-Si [7]. In contrast to $\mathrm{Si}$, however, bc8-Ge is not kinetically stable and undergoes a temperature-induced transition to hd-Ge at room temperature $[6,25]$. This results in slightly different phase compositions just upon reaching ambient pressure, as shown Fig. 1(d). In the very fast case, $\mathrm{r} 8-\mathrm{Ge}$ has not fully transformed to bc8-Ge, as indicated by the shoulder to the (211) bc8 peak (arrowed). In the intermediate case, the less pronounced shoulder suggests less $\mathrm{r} 8 \mathrm{-Ge}$. The bc8-Ge (and remaining (a)

(b)
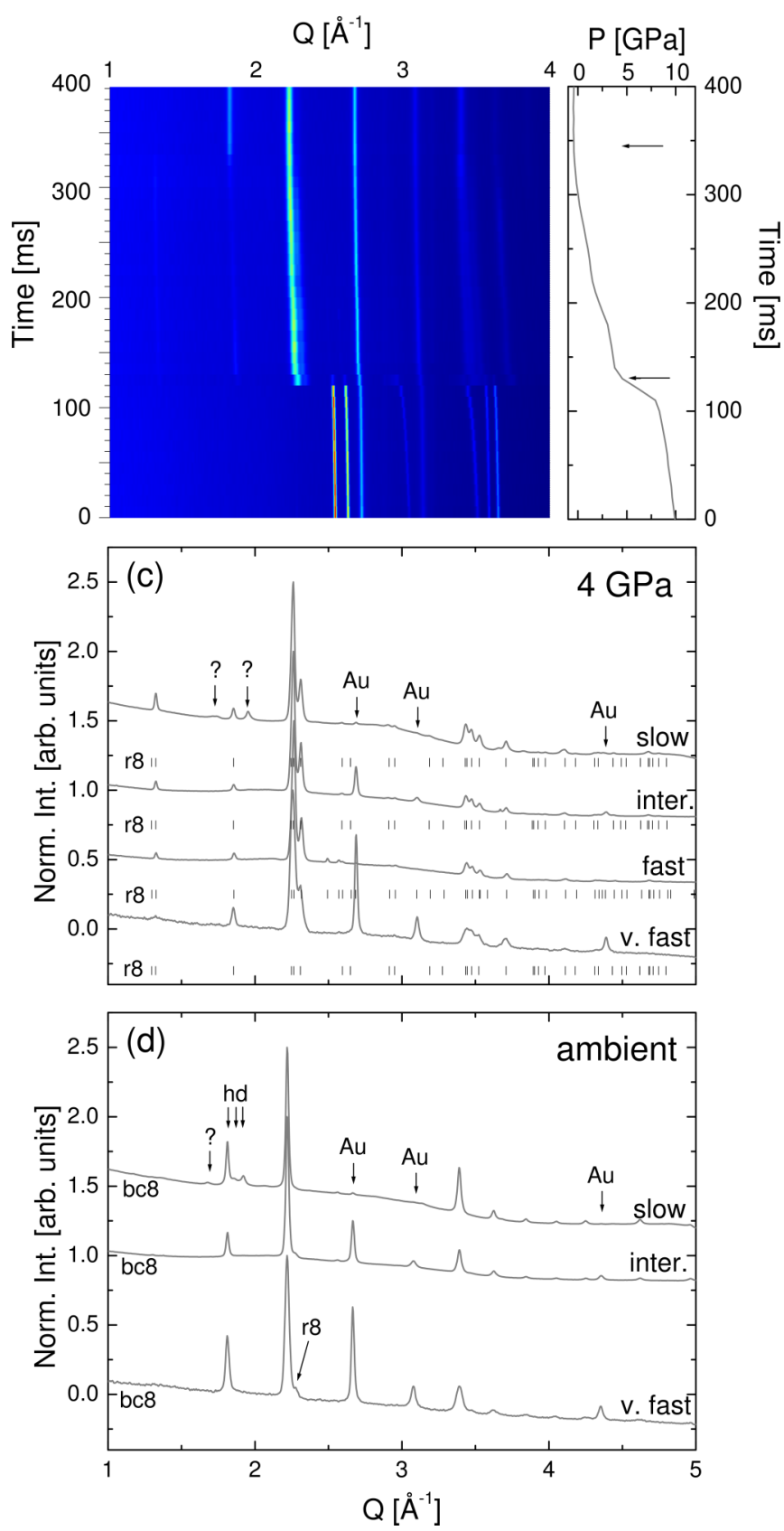

FIG. 1. (Color online) (a) Intensity contour plot of all 1D spectra taken during very fast unloading. The phase evolution with time is shown as a function of $Q$. (b) The pressure dependence on time. Spectra at $\sim 4 \mathrm{GPa}$ and ambient conditions are indicated with arrows. (c) and (d) 1D x-ray diffraction spectra for the different unloading rates at $\sim 4 \mathrm{GPa}$ and upon reaching ambient conditions. Additional phases and elements are marked with arrows and the $r 8$ peak positions from refinement are indicated by tick marks. The intensities of all spectra were normalized to 1 for the dominant $(11 \overline{2}) /(20 \overline{1}) \mathrm{r} 8$ peak and (211) bc 8 peak. For increasing times, the spectra are offset by 0.5 for clarity.

r8-Ge) decays within seconds until only hd-Ge is present. In contrast, for slow unloading no remanent r8-Ge, but instead hd-Ge (also arrowed), is present. Therefore, no pure bc8-Ge spectrum (without contribution of r8-Ge or hd-Ge) 


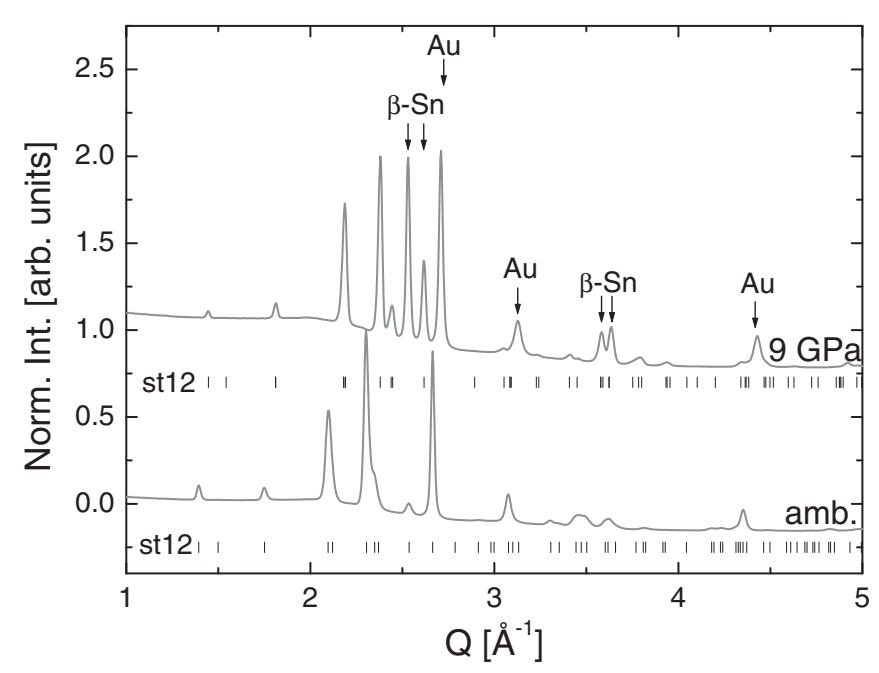

FIG. 2. 1D X-ray diffraction spectra just after the formation of st12-Ge and upon reaching ambient conditions. Additional phases and elements are marked with arrows, while the st12 peak positions from refinement are indicated by tick marks. The intensities of the spectra were normalized to 1 for the (201) peak and the spectrum at $9 \mathrm{GPa}$ was offset by 1 for clarity.

was captured. Due to the overlap of the (211) bc8 peak with the $(2 \overline{1} \overline{1}) /(20 \overline{1}) \mathrm{r} 8$ peaks and a similar overlap of the (200) and (321) bc8 peaks with the (100) and (103) hd peaks, no Rietveld refinement was performed.

Thus, subtle differences along this $\mathrm{r} 8 / \mathrm{bc} 8 / \mathrm{hd}$ transition pathway are observed. Namely, hd-Ge can form already during decompression, provided unloading is slow enough to allow the kinetically hindered transition to occur. Moreover, the $(\beta-\mathrm{Sn})-\mathrm{Ge}$ to $\mathrm{r} 8-\mathrm{Ge}$ transition shifts from 6 to $8 \mathrm{GPa}$ and the r8-Ge to bc8-Ge transition from 0.2 to $0.7 \mathrm{GPa}$ with increasing unloading time. Indeed, the stability of r8-Ge appears to increase from 6.2 to $7.6 \mathrm{GPa}$ as unloading time increases, presumably due to kinetic effects. Nonetheless, the transition pathway is fully rate independent insofar as the same $\mathrm{r} 8 / \mathrm{bc} 8 / \mathrm{hd}$ phases are always observed.

Note that for slow unloading, two peaks at $d=3.76$ and $3.29 \AA$ (indicated in Fig. 1) are observed. Their origin remains unclear since no known Ge phase (st12, r8, bc8, hd, or dc) matches. They are unlikely to be contamination, since they formed $0.2 \mathrm{GPa}$ prior to the formation of $\mathrm{r} 8 \mathrm{-Ge}$ and were not present upon loading or in the $(\beta-\mathrm{Sn})$-Ge regime. It also appears unlikely that they significantly influenced the phase behavior on slow unloading, since their volume (i.e., intensity) remained constant.

In contrast to quasihydrostatic conditions, decompression without a pressure medium yields the st 12 phase from $9 \mathrm{GPa}$ downward. As pressure is further released, the relative amount of $(\beta-\mathrm{Sn})$-Ge decreases until full transformation to st12-Ge occurs at $4 \mathrm{GPa}$, which then remains down to ambient conditions. 1D spectra from this run at $9 \mathrm{GPa}$ and ambient pressure are shown in Fig. 2. LeBail refinements were performed on both spectra, which exhibited strong preferred orientation. At $9 \mathrm{GPa}$, the refinement yields $(\beta-\mathrm{Sn})-\mathrm{Ge}$ with $a=4.964 \AA$ and $c=2.743 \AA$ and st $12-$ Ge with $a=5.760 \AA$ and $c=6.612 \AA$. After full decompression, refinement yields
st12-Ge with $a=5.926 \AA$ and $c=6.948 \AA$. This represents a small shift in $c / a$ ratio from 1.15 to 1.17 , accounting for the splitting of the (201) peak.

\section{DISCUSSION}

Our study shows that the critical parameter dominating the transition pathway is the local stress environment. In all quasihydrostatic cases, $(\beta$-Sn)-Ge transforms into the intermediate r8-Ge on decompression, as confirmed by Rietveld refinement. This is followed by bc $8-\mathrm{Ge}$ at $\sim 0.5 \mathrm{GPa}$ upon further pressure release. This phase, in turn, transforms over time as expected to hd-Ge $[4,6,21]$. In contrast, the st12 phase is formed in the presence of shear, whereby a small degree of shear is sufficient. In one additional DAC experiment (not shown) several pieces of compressed dc-Ge powder were loaded with $\mathrm{Ne}$ as the pressure medium. In this case, the Ge bridged the diamonds, also resulting in st12-Ge upon decompression. Interestingly, st12-Ge is present together with $(\beta-\mathrm{Sn})-\mathrm{Ge}$ over an extended pressure regime. This initial presence of $(\beta-\mathrm{Sn})-\mathrm{Ge}$ appears to give rise to a different $c / a$ ratio for st $12-\mathrm{Ge}$ as obtained from refinement and thus presents possible further evidence of the influence of strain on the formation of st12-Ge. In contrast, the formation of r8-Ge is sharp and sudden and its nucleation commences at $\sim 7 \mathrm{GPa}, \sim 2 \mathrm{GPa}$ lower than the nonhydrostatic st12-Ge formation. Thus, only if the nucleation of st12-Ge is avoided through the best possible quasihydrostatic conditions can $\mathrm{Ge}$ follow its hydrostatic r8/bc8 pathway.

These experimental findings shed light on previous reports on metastable Ge polymorphs. Our quasihydrostatic conditions were enabled through the use of a micron-sized beam (allowing very small sample sizes and avoiding bridging of diamonds) in combination with gas loading. This is distinctly different to earlier studies with significantly larger beam sizes $[1,6,20]$ and hence larger sample volumes. Under these conditions, bridging was clearly unavoidable, resulting in non-negligible shear. Thus, the st12 phase was nucleated in all these studies at slow, room temperature unloading [1,6,20]. However, in a nonhydrostatic environment very fast unloading (in under $0.1 \mathrm{~s}$ ) could bypass the nucleation of st12-Ge, thus yielding bc8-Ge upon full decompression, as observed in Ref. [6]. Similarly, decompression at lower temperatures may suppress the nucleation of st12-Ge, also yielding bc8-Ge $[3,23,24]$. Moreover, the use of amorphous Ge [22,25] may have limited the microstrain between $(\beta$-Sn)-Ge crystals, thus reducing shear and giving rise to a higher likelihood for the $\mathrm{r} 8 / \mathrm{bc} 8$ phases. Hence, a dependence on hydrostaticity is consistent with previous observations.

We will now examine previous reports of the $r 8$ phase in a DAC. For example, a first study gives tentative evidence for r8-Ge at low temperature [23]. This may be attributed to stabilization at lower temperatures (similarly to bc8-Ge being metastable at low temperatures). Two further reports $[18,28]$ observed r8-Ge mixed with dc-Ge after extremely slow unloading (typically over $10 \mathrm{~h}$ ). The very small beam sizes used in these two studies [18,28] potentially could allow also quasihydrostatic conditions. Since these studies used ethanol-methanol as the pressure medium (which is quite hydrostatic below $11 \mathrm{GPa}$ [36]) the formation of $\mathrm{r} 8-\mathrm{Ge}$ is hence not surprising. However, the possible formation of dc-Ge from 
$(\beta-\mathrm{Sn})-\mathrm{Ge}$ at room temperature is unexpected. We therefore suggest that the formation of $\mathrm{dc}-\mathrm{Ge}$ results from the kinetic instability of these metastable phases and is induced through temperature. A similar effect is indeed observed for the bc8-Ge to hd-Ge transition (with no hd-Ge present upon very fast unloading, but observed during slow unloading). It is thus quite possible that the extremely slow unloading of $\sim 10 \mathrm{~h}$ may provide sufficient time for the transformation of the metastable r8-Ge to the stable dc-Ge, since sufficient energy is provided to overcome the kinetic barrier between these two phases. Indeed, a transition from an $\mathrm{r} 8$ to dc structure has been observed for $\mathrm{Si}$ [37] and may hence also be expected for Ge. Therefore, these previous findings are also consistent with our proposal for a critical dependence on the hydrostaticity.

The literature is now reviewed for $a b$ initio modeling of these transitions [12,14,26,38-40]. Calculations of enthalpypressure curves within density functional theory (DFT) provide additional insights through the prediction of transition pathways and pressures. For the metastable regime of $\mathrm{Ge}$, however, no conclusive answer is yielded since some report the st12 phase as energetically more stable [38,40], while others favor the bc 8 structure [12]. Although less work has been done on r8-Ge [12,14], a strong dependence on modeling parameters is apparent. Indeed, recent work predicts a transition sequence of $(\beta-\mathrm{Sn})-\mathrm{Ge}$ to st12 to $\mathrm{r} 8$ to bc8 when using the local density approximation (LDA) [12]. However, $(\beta-\mathrm{Sn})-\mathrm{Ge}$ to st12 to bc8 is observed when the generalized gradient approximation (GGA) is used and the stability regime of r8-Ge disappears [12]. This dependence on the functional, however, cannot be equated with dependence on shear. Therefore, a combination of molecular dynamics and density functional tight binding theory (as has been conducted for the r8-Ge to bc8-Ge transition [39]) may be advantageous. Furthermore, current calculations assume hydrostatic conditions and shear is not taken into account. Therefore, these avenues will be pursued, but are outside the scope of this paper.

Although sole consideration of coexistence pressures from enthalpy-pressure curves is not sufficient for the determination of transition pathways, it can give accurate transition pressures. We included the experimental transition pathways and Ge polymorphs (r8, bc8, and st12) into our calculations and obtained very good agreement between predictions and experiment. Namely, using LDA, the transition of ( $\beta$-Sn)-Ge to r8-Ge is predicted at $6.4 \mathrm{GPa}$ (experimentally at $\sim 7 \mathrm{GPa}$ ), followed by the r8-Ge to bc8-Ge transition at $0.65 \mathrm{GPa}$ (experimentally at $\sim 0.5 \mathrm{GPa})$. In contrast, the $(\beta-\mathrm{Sn})$-Ge to st12-Ge transition occurs at higher pressures, at $6.8 \mathrm{GPa}$ with LDA and at $8.4 \mathrm{GPa}$ with GGA (experimentally at $\sim 9 \mathrm{GPa}$ ).

Based on our results, it is now clear that a simple change of hydrostaticity enables control of the metastable Ge polymorph formed. Such control is necessary for any potential technological applications. For example, st12-Ge, metastable at ambient conditions [1,21], is now simply formed in a DAC or also through uniaxial indentation loading with pointed tips (yielding higher shear) [41]. Interestingly, this structure may exhibit superconducting properties possibly accessible through doping [11]. Along the other transition pathway, hd-Ge is the metastable end polymorph [21,42] and can now be reliably formed in both a DAC and also via indentation [42]. A direct band gap of $0.55 \mathrm{eV}$ has theoretically been predicted for this structure $[12,43]$, which is potentially useful.

In contrast, the precursors to hd-Ge, bc8-Ge, and r8-Ge are only kinetically stable at lower temperatures $[3,26]$. It may, however, be possible to stabilize these polymorphs at room temperature through doping, making their technological exploitation more practicable. For example, bc8-Ge is, in contrast to the other polymorphs, most likely semimetallic $[12,43]$. Furthermore, the r8 phase may become practically accessible due to its kinetic stability down to $0.5 \mathrm{GPa}$ in a DAC and its kinetic stability at dry ice temperature if created by indentation [26]. The electrical properties of this polymorph remain unclear since no experimental studies have been conducted and DFT modeling is known to underestimate the band gap [12]. For Si, however, superior optical absorption properties have been predicted for the r8 structure [10]. Similar properties for $\mathrm{r} 8$-Ge could consequently also make it an interesting material for photovoltaic applications.

The use of shear as a parameter for the controlled formation of desired polymorphs may also have implications for other group IV elements or compound semiconductors [17]. Few data exist from DAC experiments on the influence of shear on the metastable regime, although it is well known that nonhydrostatic conditions lower transition pressures upon compression (see Ref. [17] and references therein). Interestingly, recent $a b$ initio modeling on a different tetrahedral system, silica, indicated that shear during compression can enable the formation of phases energetically unfavorable under hydrostatic conditions, thus giving rise to entirely different structures [44-46].

In addition to these experimental and $a b$ initio results, observations from indentation experiments give complementary insight. It has been clear that indentation of dc-Ge with pointed tips (resulting in higher shear) can produce metastable phases [47], while "standard" indentation with spherical tips (resulting in more hydrostatic conditions) yields no phase transition, but plastic deformation via dislocations and twinning [48]. This defect formation can be suppressed through rapid loading and consequently metastable polymorphs form upon decompression [49]. Comparisons of recent DFT calculations of Raman modes [26] with the experimental Raman data from this study [49] as well as the presented transmission electron diffraction data [49] clearly indicate the presence of st12-Ge in this case. Interestingly, such rapid indentation loading results in significant fracturing of the surrounding dc-Ge [49] and thus high shear stresses. These findings are therefore consistent with our DAC findings. Furthermore, the formation of dislocations can also be prevented through the use of pure amorphous $\mathrm{Ge}$ as the starting material. In this case, more hydrostatic indentation loading (with a large spherical tip, but without fracturing) results in the formation of r8-Ge without any evidence of st12-Ge [26]. Thus, indentation reveals the same shear dependence for Ge, with hydrostatic conditions resulting in $\mathrm{r} 8-\mathrm{Ge}$, but less hydrostatic conditions in st12-Ge [41].

Such examples can also be found for other materials. In the case of Si, fast unloading in a DAC results in (not fully identified) crystalline structures [5], but fast indentation unloading (with higher shear) in pressure-induced amorphization [50]. Furthermore, an example may also be found for a compound 
semiconductor, InSb. In this case indentation with a pointed tip yields a metastable polymorph that cannot be easily matched to known (more hydrostatically obtained) phases [47].

Thus, these observations suggest that exploiting quasihydrostatic versus nonhydrostatic conditions opens exciting avenues for the synthesis of metastable phases. Our finding may, therefore, have considerable impact not only for the high pressure synthesis of different structures from the known semiconductor materials, but also for other phases with technologically useful applications such as, for example, superhard or even high-temperature superconducting phases.

\section{CONCLUSION}

In conclusion, we find that the formation of metastable $\mathrm{Ge}$ polymorphs can be controlled through variations in hydrostaticity. While quasihydrostatic conditions yield transitions along the $\mathrm{r} 8 / \mathrm{bc} 8 / \mathrm{hd}$ pathway, the presence of shear results in st12-Ge. This control now allows for reliable formation of different Ge polymorphs, which may facilitate their technological exploitation. Furthermore, our results suggest avenues for the formation of different metastable phases also in other materials.

\section{ACKNOWLEDGMENTS}

Work by M.G. was fully supported by EFree, an Energy Frontier Research Center funded by the U.S. Department of Energy, Office of Science, Basic Energy Sciences under Award No. DE-SC0001057. M.L.C. was supported by NSF Grant No. DMR-IO-1006184 and the theory program at the Lawrence Berkeley National Laboratory through the Office of Basic Energy Science, U.S. Department of Energy under Contract No. DE-AC02-05CH11231. J.E.B. is supported by an ARC QEII fellowship. This work was performed at HPCAT (Sector 16), Advanced Photon Source (APS), Argonne National Laboratory. HPCAT operations are supported by DOE-NNSA under Award No. DE-NA0001974 and DOE-BES under Award No. DE-FG02-99ER45775, with partial instrumentation funding by NSF. Use of the COMPRES-GSECARS gas loading system was supported by COMPRES under NSF Cooperative Agreement No. EAR 11-57758 and by GSECARS through NSF Grant No. EAR-1128799 and DOE Grant No. DEFG02-94ER14466. A.P.S. is supported by DOE-BES, under Contract No. DE-AC02-06CH11357. The authors gratefully acknowledge funding from the Australian Research Council (ARC).
[1] F. P. Bundy and J. S. Kasper, Science 139, 340 (1963).

[2] R. H. Wentorf Jr. and J. S. Kasper, Science 139, 338 (1963).

[3] C. H. Bates, F. Dachille, and R. Roy, Science 147, 860 (1965).

[4] J. S. Kasper and J. R. H. Wentorf, Science 197, 599 (1977).

[5] Y.-X. Zhao, F. Buehler, J. R. Sites, and I. L. Spain, Solid State Commun. 59, 679 (1986).

[6] R. J. Nelmes, M. I. McMahon, N. G. Wright, D. R. Allan, and J. S. Loveday, Phys. Rev. B 48, 9883 (1993).

[7] R. O. Piltz, J. R. Maclean, S. J. Clark, G. J. Ackland, P. D. Hatton, and J. Crain, Phys. Rev. B 52, 4072 (1995).

[8] D. Ge, V. Domnich, and Y. Gogotsi, J. Appl. Phys. 95, 2725 (2004).

[9] G. Weill, J. L. Mansot, G. Sagon, C. Carlone, and J. M. Besson, Semicond. Sci. Technol. 4, 280 (1989).

[10] B. D. Malone, J. D. Sau, and M. L. Cohen, Phys. Rev. B 78, 161202(R) (2008).

[11] B. D. Malone, J. D. Sau, and M. L. Cohen, Phys. Rev. B 78, 035210 (2008).

[12] B. D. Malone and M. L. Cohen, Phys. Rev. B 86, 054101 (2012).

[13] S. Botti, J. A. Flores-Livas, M. Amsler, S. Goedecker, and M. A. L. Marques, Phys. Rev. B 86, 121204(R) (2012).

[14] D. Selli, I. A. Baburin, R. Martoňák and S. Leoni, Sci. Rep. 3, 1466 (2013).

[15] J. C. Jamieson, Science 139, 762 (1963).

[16] S. Minomura and H. G. Drickamer, J. Phys. Chem. Solids 23, 451 (1962)

[17] A. Mujica, A. Rubio, A. Munõz, and R. J. Needs, Rev. Mod. Phys. 75, 863 (2003).

[18] A. Wosylus, Y. Prots, W. Schnelle, M. Hanfland, and U. Schwarz, Z. Naturforsch. 63b, 608 (2008).

[19] Note that instead of the historical numbering for consistency with literature but improved ease of understanding, acronyms are used for the dc and hd structure, symmetry descriptions for the other metastable phases, i.e., bc8, r8, and st12, and bracketed writing for reference elements, i.e., $(\beta$-Sn).

[20] J. S. Kasper and S. M. Richards, Acta Crystallogr. 17, 752 (1964).

[21] V. V. Brazhkin, A. G. Lyapin, S. V. Popova, and R. N. Voloshin, Pis'ma Zh. Eksp. Teor. Fiz. 56, 156 (1992) [JETP Lett. 56, 152 (1992)].

[22] M. Imai, T. Mitamura, K. Yaoita, and K. Tsuji, High Press. Res. 15, 167 (1996).

[23] A. G. Lyapin, V. V. Brazhkin, S. V. Popova, and A. V. Sapeliki, Phys. Status Solidi B 198, 481 (1996).

[24] V. V. Brazhkin, A. G. Lyapin, S. V. Popova, and R. N. Voloshin, Phys. Rev. B 51, 7549 (1995).

[25] S. Minomura, J. Phys. Colloq. 42, C4-181 (1981).

[26] B. C. Johnson, B. Haberl, S. Deshmukh, B. D. Malone, M. L. Cohen, J. C. McCallum, J. S. Williams, and J. E. Bradby, Phys. Rev. Lett. 110, 085502 (2013).

[27] F. Coppari, J.C. Chervin, A. Congeduti, M. Lazzeri, A. Polian, E. Principi, and A. Di Cicco, Phys. Rev. B 80, 115213 (2009).

[28] U. Schwarz, A. Wosylus, B. Böhme, M. Baitinger, M. Hanfland, and Y. Grin, Angew. Chem., Int. Ed. Engl. 47, 6790 (2008).

[29] S. V. Sinogeikin et al. (unpublished).

[30] A. P. Hammersley, S. O. Svensson, M. Hanfland, A. N. Fitch, and D. Häusermann, High Press. Res. 14, 235 (1996).

[31] A. P. Hammersley, ESRF, Internal Report No. ESRF97HA02T, 1997 (unpublished).

[32] O. L. Anderson, D. G. Isaak, and S. Yamamoto, J. Appl. Phys. 65, 1534 (1989).

[33] B. H. Toby, J. Appl. Crystallogr. 34, 210 (2001).

[34] A. C. Larson and R. B. V. Dreele, Los Alamos National Laboratory, Report No. LAUR, 1994, pp. 86-748.

[35] A. Le Bail, Powder Diffr. 20, 316 (2005). 
[36] S. Klotz, J.-C. Chervin, P. Munsch, and G. L. Marchand, J. Phys. D: Appl. Phys. 42, 075413 (2009).

[37] B. Haberl, M. Guthrie, S. V. Sinogeikin, G. Shen, J. S. Williams, and J. E. Bradby (unpublished).

[38] A. Mujica and R. J. Needs, Phys. Rev. B 48, 17010 (1993).

[39] D. Selli, S. E. Boulfelfel, I. A. Baburin, G. Seiferta, and S. Leoni, RSC Adv. 2, 8833 (2012).

[40] J.-T. Wang, C. Chen, H. Mizuseki, and Y. Kawazoe, Phys. Rev. Lett. 110, 165503 (2013).

[41] S. Deshmukh, B. Haberl, S. Ruffell, P. Munroe, J. S. Williams, and J. E. Bradby, J. Appl. Phys. 115, 153502 (2014).

[42] J. S. Williams, B. Haberl, S. Deshmukh, B. C. Johnson, B. D. Malone, M. L. Cohen, and J. E. Bradby, Phys. Status Solidi RRL 7, 355 (2013).
[43] J. D. Joannopoulos and M. L. Cohen, Phys. Rev. B 7, 2644 (1973).

[44] M. Durandurdu, Phys. Rev. B 80, 024102 (2009).

[45] D. Donadio, R. Martoňák, P. Raiteri, and M. Parrinello, Phys. Rev. Lett. 100, 165502 (2008).

[46] Y. Liang, C. R. Miranda, and S. Scandolo, Phys. Rev. Lett. 99, 215504 (2007).

[47] A. Kailer, K. G. Nickel, and Y. G. Gogotsi, J. Raman Spectrosc. 30, 939 (1999).

[48] J. E. Bradby, J. S. Williams, J. Wong-Leung, M. V. Swain, and P. Munroe, Appl. Phys. Lett. 80, 2651 (2002)

[49] D. J. Oliver, J. E. Bradby, J. S. Williams, M. V. Swain, and P. Munroe, J. Appl. Phys. 105, 126101 (2009).

[50] B. Haberl, A. C. Y. Liu, J. E. Bradby, S. Ruffell, J. S. Williams, and P. Munroe, Phys. Rev. B 79, 155209 (2009). 